\title{
BMJ Open Validation of days at home as an outcome measure after surgery: a prospective cohort study in Australia
}

\author{
Paul S Myles, ${ }^{1,2,3}$ Mark A Shulman, ${ }^{1}$ Stephane Heritier, ${ }^{3}$ Sophie Wallace, ${ }^{1}$ \\ David R Mcllroy, ${ }^{1,2}$ Stuart McCluskey, ${ }^{4}$ Isabella Sillar, ${ }^{2}$ Andrew Forbes ${ }^{3}$
}

To cite: Myles PS, Shulman MA, Heritier S, et al. Validation of days at home as an outcome measure after surgery: a prospective cohort study in Australia. BMJ Open 2017;7:e015828. doi:10.1136/ bmjopen-2017-015828

- Prepublication history and additional material are available. To view please visit the journal online (http://dx.doi.org/10. 1136/bmjopen-2017-015828).

Received 3 January 2017 Revised 14 June 2017 Accepted 19 June 2017

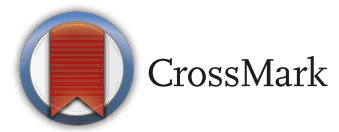

${ }^{1}$ Department of Anaesthesia and Perioperative Medicine, Alfred Hospital, Melbourne, Victoria, Australia

${ }^{2}$ Department of Anaesthesia and Perioperative Medicine, Monash University, Melbourne, Victoria, Australia

${ }^{3}$ Department of Epidemiology and Preventive Medicine,

Monash University, Melbourne, Victoria, Australia

${ }^{4}$ Toronto General Hospital and University of Toronto, Toronto, Ontario, Canada

Correspondence to Professor Paul S Myles; p.myles@alfred.org.au

\section{ABSTRACT}

Objective To evaluate 'days at home up to 30 days after surgery' $\left(\mathrm{DAH}_{30}\right)$ as a patient-centred outcome measure. Design Prospective cohort study.

Data source Using clinical trial data (seven trials, 2109 patients) we calculated $\mathrm{DAH}_{30}$ from length of stay, readmission, discharge destination and death up to 30 days after surgery.

Main outcome The association between $\mathrm{DAH}_{30}$ and serious complications after surgery.

Results One or more complications occurred in 263 of $1846(14.2 \%)$ patients, including 19 (1.0\%) deaths within 30 days of surgery; $245(11.6 \%)$ patients were discharged to a rehabilitation facility and $150(7.1 \%)$ were readmitted to hospital within 30 days of surgery. The median $\mathrm{DAH}_{30}$ was significantly less in older patients $(p<0.001)$, those with poorer physical functioning $(p<0.001)$ and in those undergoing longer operations $(p<0.001)$. Patients with serious complications had less days at home than patients without serious complications $(20.5(95 \% \mathrm{Cl} 19.1$ to 21.9$)$ vs 23.9 (95\% Cl 23.8 to 23.9$) p<0.001)$, and had higher rates of readmission $(16.0 \%$ vs $5.9 \% ; p<0.001)$. After adjusting for patient age, sex, physical status and duration of surgery, the occurrence of postoperative complications was associated with fewer days at home after surgery (difference 3.0(95\% Cl 2.1 to 4.0) days; $p<0.001)$. Conclusions $\mathrm{DAH}_{30}$ has construct validity and is a readily obtainable generic patient-centred outcome measure. It is a pragmatic outcome measure for perioperative clinical trials.

\section{INTRODUCTION}

Surgery and other interventional procedures are intended to relieve symptoms and in many cases prolong life. But surgery is not risk free; perioperative complications can impair patient recovery resulting in prolonged hospitalisation, short or longer term disability and sometimes poor survival. A wide variety of outcome measures have been used to quantify each of these aspects of the postoperative experience but few provide a broad, patient-centred perspective of effective and efficient care ${ }^{1}$; these are needed to better inform the current shift towards valuebased healthcare. ${ }^{23}$

\section{Strengths and limitations of this study}

This study integrates length of stay, readmission, discharge destination and early deaths after surgery into a single outcome metric, 'days at home up to 30 days after surgery' $\left(\mathrm{DAH}_{30}\right)$.

- Patients hope to recover quickly after surgery, free of complications and need for readmission; $\mathrm{DAH}_{30}$ is thus a patient-centred outcome.

- Accurate calculation of $\mathrm{DAH}_{30}$ requires knowledge of postdischarge location (home or nursing facility) and any readmissions at the index or other hospitals.

- Because early deaths heavily influence the $\mathrm{DAH}_{30}$ metric, this information should be additionally reported if, say, the incidence exceeds $10 \%$.

Patient-centred care requires clinicians to consider outcomes that matter most to patients. That is, the patient's experience of their illness, quality of life and functioning; their values, preferences and goals for healthcare. ${ }^{4}$ Loss of the ability to live independently is a major concern for the elderly ${ }^{5}$; it is clearly a patient-centred outcome, and has been associated with postoperative readmissions and death after hospital discharge. ${ }^{5}$

Specific periprocedural complications such as surgical site infection, respiratory failure, delirium and myocardial infarction are clearly important to patients and physicians alike, but reliable and consistent detection is problematic. In any case such information is an incomplete description of the overall success of surgery and other perioperative care, and does not describe the impact of such complications on functioning and need for institutionalisation. Similar challenges occur when nominating endpoints in clinical trials, including a lack of standardisation, ${ }^{7}$ need for adjudication, and uncertainty about the overall health impact of each endpoint on a patient's recovery. There is a growing acceptance that outcome measures used in clinical trials should be determined in partnership 
by patients and physician-researchers, aiming to identify outcomes that are important to patients. ${ }^{8}$

'Days alive and out of hospital' has been shown to be a readily quantifiable and patient-centred outcome measure in some chronic cardiovascular conditions such as heart failure and atrial fibrillation, ${ }^{9-11}$ and in geriatric medicine, ${ }^{12}$ but it has not been used as an outcome measure in perioperative trials. Home discharge has been proposed as a proxy for a patient's recovery after surgery, ${ }^{13}$ and is estimated when using the American College of Surgeons' Surgical Risk Calculator, ${ }^{14}$ but this does not account for readmissions or early deaths, although the latter collects and reports some of this information. ${ }^{15}$

Our own work and that of others have shown that early return home after surgery ${ }^{6}{ }^{16-18}$ and medical illnesses such as stroke ${ }^{1920}$ are highly valued by patients but could be undermined if the patient were to be transferred to another type of nursing facility. A more favourable perioperative outcome measure should account for both the initial hospital stay associated with the index surgery, rehospitalisation due to postdischarge complications, discharge to institutional care and early deaths.

We thus chose to evaluate the utility of 'days (alive and) at home' within 30 days of surgery $\left(\mathrm{DAH}_{30}\right)$ in the surgical/perioperative setting as a patient-centred outcome measure for perioperative clinical trials and quality improvement activities. Our hypothesis was that $\mathrm{DAH}_{30}$ would be lower in higher risk patients, those undergoing more extensive surgery and in those with complications after surgery (ie, it has construct validity).

\section{METHODS}

This manuscript was written in adherence to the STROBE (Strengthening the Reporting of Observational Studies in Epidemiology) statement. ${ }^{21}$

\section{Study design and data sources}

Data were obtained from each of seven recently completed clinical trials that prospectively enrolled patients undergoing various types of elective and emergency surgery at the Alfred Hospital in Melbourne, Australia. The cohort consisted of four multicentre randomised trials and three before-and-after studies (see online supplementary table S1) ${ }^{22-29}$ For each trial, we collected a comparable set of patient demographic and perioperative characteristics, and clinical outcome measures, including complete hospital discharge, discharge destination (home, rehabilitation facility, nursing home) and readmission data. All but one study ${ }^{28}$ prospectively recorded readmission data; for the latter study, we could obtain this information retrospectively from our hospital information system. Both the present study and each of the original trials received institutional ethics committee approval.

\section{Patients}

Patients 18 years and older undergoing an elective or non-elective inpatient operation enrolled in one of the aforementioned trials were included. Study inclusion criteria were established for the original studies and typically identified those at increased risk of postoperative complications. In all cases, patients provided informed consent before enrolment in the original trials.

\section{Patient involvement}

Hospital patients have previously indicated the importance of returning home after hospitalisation for medical or surgical conditions, ${ }^{616-20}$ but we did not involve patients or their carers in the design or conduct of this study.

\section{Risk factors and outcomes}

Perioperative data included patient demographics, comorbidity, functional status, type and duration of surgery, hospital length of stay, hospital readmission(s), and in all but one study ${ }^{28}$ we prospectively collected selected complications at 30 days after surgery: wound infection, myocardial infarction, stroke, pulmonary embolism, cardiac arrest and death.

Hospital discharge data were used to calculate hospital length of stay. Whether the patient was discharged from hospital to their home or to a nursing facility was obtained from the electronic medical record, but for those admitted to a rehabilitation facility we were unable to ascertain the number of days admitted before eventual discharge home. For those readmitted to hospital, we combined the original length of stay with subsequent hospital stay(s) to calculate total length of stay within 30 days postoperatively.

$\mathrm{DAH}_{30}$ was calculated using mortality and hospitalisation data from the date of the index surgery (day 0 ). For example, if patients died on day 2 after their surgery, they were assigned $0 \mathrm{DAH}_{30}$, if patients were discharged from hospital on day 6 after surgery but were subsequently readmitted for 4 days before their second hospital discharge, then they were assigned $20 \mathrm{DAH}_{30}$. If patients died within 30 days of surgery, irrespective of whether they had spent some time at home, $\mathrm{DAH}_{30}$ was scored as zero (0). Further explanation is provided in the online supplementary appendix.

Patients are commonly admitted to a postacute hospital rehabilitation centre after lower limb arthroplasty and cardiac surgery in our setting; some frail and elderly patients are also transferred for ongoing convalescence. We were unable to reliably collect secondary length of stay for rehabilitation facilities-we thus did two secondary analyses, assuming the length of stay in a rehabilitation facility was 5 or 14 extra days. That is, $\mathrm{DAH}_{30 \text {-rehab5 }}$ was calculated as $\mathrm{DAH}_{30}-5$, and $\mathrm{DAH}_{30 \text {-rehab14 }}$ was calculated as $\mathrm{DAH}_{30}-14$, in secondary analyses.

For the multicentre trials, ${ }^{22-24}$ a 12-lead electrocardiograph was recorded preoperatively and on days 1 and 3 after surgery. Blood for troponin (or if unavailable, creatine kinase-myocardial band) measurement was collected at 6-12 hours after surgery and on the first three postoperative days. In all trials, laboratory tests were otherwise ordered if clinically indicated. Each complication 
was defined within the original study protocol and in all cases a consistent definition was used. In brief, surgical site infection was confirmed if associated with purulent discharge, with or without a positive microbial culture; or pathogenic organisms isolated from aseptically obtained microbial culture, ${ }^{30}$ although the most recent trial ${ }^{27}$ included documentation of a physician's diagnosis in this definition. ${ }^{31}$ Pneumonia was confirmed by a new pulmonary infiltrate reported by chest X-ray or CT, in association with at least one of the following: temperature $>38^{\circ} \mathrm{C}$, white cell count $>12000 / \mathrm{mL}$, or positive sputum culture that was not heavily contaminated with oral flora or that corresponded with positive blood cultures. Myocardial infarction was defined according to the third universal definition, ${ }^{32}$ requiring elevated cardiac biomarker plus at least one of the following: (1) ischaemic symptoms, (2) pathological $Q$ waves, (3) electrocardiographic changes indicative of ischaemia, (4) coronary artery intervention or (5) new wall motion abnormality on echocardiography or scanning; or autopsy finding of myocardial infarction. The threshold for significant elevated troponin was the hospital laboratory's 99th percentile of a normal reference population (upper reference limit), according to recent recommendations. ${ }^{33}$ Stroke was confirmed if a new neurological deficit is persisting for at least 24 hours, verified by neurologist assessment and/or CT or MRI.

\section{Statistical analysis}

Data were first merged and checked for inconsistencies. Patient age was grouped into 10-year categories, and hourly cut-points for duration of surgery (2, 3 and 4 hours) were created to generate approximately similar group sizes and facilitate clinical interpretation. $\mathrm{DAH}_{30}$ was analysed using quantile regression. ${ }^{34}$ This approach, well known in econometrics where it was initially introduced, allows the modelling of any quantile of a continuous endpoint, here $\mathrm{DAH}_{30}$, as a linear combination of the covariates. As $\mathrm{DAH}_{30}$ is left skewed with a spike at zero, it is more relevant to model the median (or alternatively, the 75th percentile) that is closer to the major distribution mode and directly interpretable. The choice of the quantile(s) to be analysed can be prespecified or a range of values selected for their meaningfulness or exploratory purposes. Here the range 50th-75th percentile was deemed relevant. No assumption on the true distribution of the endpoint is required. The asymptotic distribution of the parameter estimates can be derived but depends on some unknown density estimate. In general, resampling methods are recommended to obtain CIs. ${ }^{35}{ }^{36}$ Raw and adjusted medians and their 95\% CIs obtained by bootstrapping as implemented in Stata with 1000 replicates were reported for key predictors. The adjusted models included age by 10-year categories, sex, American Society of Anesthesiologists (ASA) physical status score and surgery time ( $<2$ hours, $2.0-2.99,3.0-3.99, \geq 4.0)$. A goodness-of-fit test ${ }^{37}$ comparing this model to the full model including the same predictors plus smoking, heart failure and diabetes was not any better $(\mathrm{p}=0.36)$. A global test of effect of any key predictor was carried out using a quasilikelihood ratio test. ${ }^{37}$ Quantile regression was also used to test median differences between those with and without complications, and by postoperative complications. Supplementary analyses were done for the 75th percentile (Q3). All analyses were done using Stata V.14.0 except the quasilikelihood ratio test analysis that is only available in SAS v9.4. All tests were two sided and performed at level $\mathrm{p}=0.05$; no correction was made for multiple comparisons.

\section{RESULTS}

A total of 2109 eligible patients 18 years and older were enrolled into clinical trials and underwent inpatient operations at the Alfred Hospital between March 2006 and September 2016. The number of patients enrolled in each of the trials is detailed in the online supplementary table S1. The cohort included 1427 male patients (67.7\%) with a mean (SD) age of 65 (12) years who underwent a range of inpatient operations (table 1). Most operations were cardiac surgical procedures $(679(32.2 \%))$, followed by general $(489(23.2 \%))$, urological $(315(14.9 \%))$ and neurosurgical procedures $(220(10.4 \%))$.

There was a bimodal, skewed distribution of $\mathrm{DAH}_{30}$ (figure 1). The spike at zero consisted of 19 patients $(1.0 \%)$ who died, and 40 patients remaining in hospital at least 30 days after surgery. $\mathrm{DAH}_{30}$ and rates of admission to a rehabilitation centre varied according to type of surgery (table 2 ).

One or more complications occurred in 263 (14.2\%) patients. Overall, 245 (11.6\%) patients were admitted to a rehabilitation facility and $150(7.1 \%)$ were readmitted within 30 days of surgery. The median $\mathrm{DAH}_{30}$ was 23.7 (95\% CI, 23.5 to 24.0 ), but this varied according to type of surgery (table 1).

The median $\mathrm{DAH}_{30}$ was significantly less in older patients, current smokers, diabetics, those with poorer physical functioning and undergoing longer operations (table 2). These associations remained after adjustment for all of these covariates and patient sex (table 2). The individual complications of myocardial infarction, stroke, pulmonary embolism and surgical site infection were each associated with shorter $\mathrm{DAH}_{30}$ (table 3 ) in a raw analysis. Hospital readmission was also a factor, decreasing median $\mathrm{DAH}_{30}$ when compared with those not readmitted to hospital, 17.9 (95\% CI 16.3 to 19.5) vs 23.9 (95\% CI 23.8 to 23.9$)$, respectively $(\mathrm{p}<0.0001)$.

After adjusting for patient age, sex, ASA physical status and duration of surgery, the occurrence of any postoperative complication was associated with fewer days at home after surgery (difference 3.0 (95\% CI 2.1 to 4.0) days; $\mathrm{p}<0.0001)$.

\section{Supplementary analyses}

The above findings were consistent when analysing the third quartile distributions and differences (online supplementary tables S2-S4 in the appendix), 
Table $1 \mathrm{DAH}_{30}$ according to types of surgery

\begin{tabular}{llcl}
\hline Surgery & Number of patients & $\begin{array}{l}\text { Number admitted to a } \\
\text { rehabilitation hospital (\%) }\end{array}$ & Median (95\% $\mathbf{C l}_{\text { DAH }}{ }^{*}{ }^{*}$ \\
\hline Cardiac & 679 & $54(8.0)$ & $22.8(22.6$ to 22.9$)$ \\
Orthopaedic & 289 & $122(42)$ & $21.9(21.2$ to 22.6$)$ \\
Neurosurgery & 220 & $9(4.0)$ & $22.8(22.2$ to 23.5$)$ \\
Colorectal & 118 & $8(6.8)$ & $24.9(23.9$ to 26.0$)$ \\
Urology & 315 & $26(8.3)$ & $23.8(23.0$ to 24.5$)$ \\
Vascular & 56 & $1(1.8)$ & 26.0 (24.3 to 27.3$)$ \\
Ear, nose, throat & 99 & $17(17)$ & $25.8(24.9$ to 27.0$)$ \\
Oesophagogastric/hepatobiliary & 253 & $4(1.6)$ & $24.9(23.8$ to 26.1$)$ \\
Thoracic & 28 & $2(7.1)$ & $22.8(17.8$ to 27.8$)$ \\
Other & 52 & $2(3.8)$ & $28.8(27.7$ to 30.0$)$ \\
\hline
\end{tabular}

*Hospital days do not include those spent in a rehabilitation facility. $\mathrm{DAH}_{30}$, days at home up to 30 days after surgery.

and after accounting for the additional loss of days at home because of admission to a rehabilitation centre (online supplementary tables S5-S9 in the appendix). The impact of the quantile (50th-75th percentile) choice for $\mathrm{DAH}_{30}$ on the associations of patient age category, ASA physical status and surgical duration was stable over the clinical ranges studied (online supplementary figure S1).

\section{DISCUSSION}

We found that $\mathrm{DAH}_{30}$ has construct validity and is a readily obtainable patient-centred outcome measure that could be used to better inform patients and physicians when planning surgery. Unlike previous related measures, $\mathrm{DAH}_{30}$ accounts for each of delayed hospital discharges because of postoperative complications, discharge to a rehabilitation centre or other postacute care nursing facility, rehospitalisations and postoperative deaths. It thus captures much of the surgical experience,

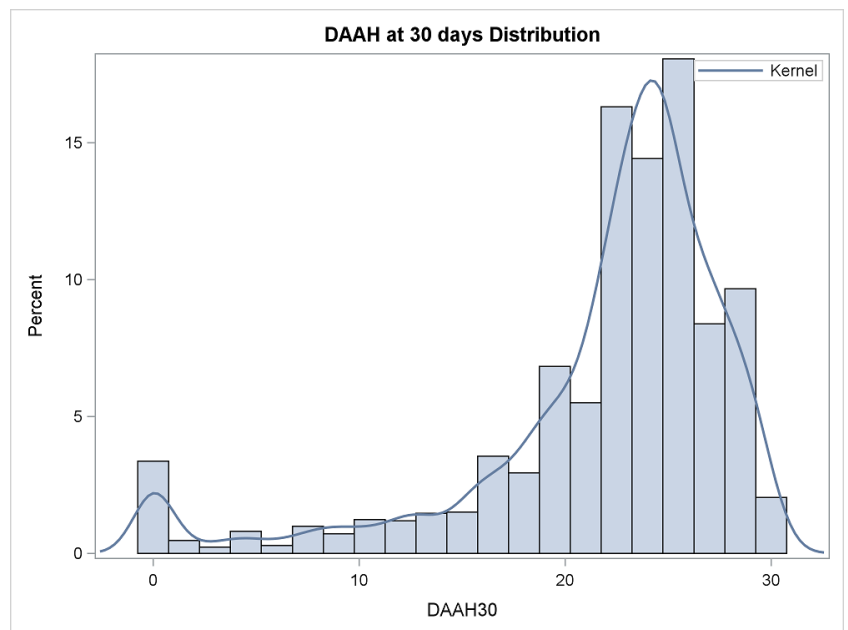

Figure 1 Frequency distribution of days alive and at home (DAAH) up to 30 days after surgery $(n=2109)$. The smoothing line (kernel) is a non-parametric estimate of the probability density function. integrating efficacy, quality and safety, and thus reflecting value-based care. It can also be risk adjusted for benchmarking purposes. $\mathrm{DAH}_{30}$ will be maximised when patients recover free of complications after surgery, with optimal comfort and functioning-aligning with patient values and preferences, and goals for healthcare. ${ }^{4}$

Although concerns are frequently raised about the usefulness of hospital length of stay as an outcome measure after surgery, largely because of social factors and reluctance to discharge on weekends, it mostly adds variance (background noise) in clinical trials and is not biased. Hospital stay is a reasonable surrogate for quality and speed of recovery after surgery, ${ }^{13}{ }^{38-40}$ and it has marked resource/cost implications. Most patients want to go home as soon as possible-it is a desired outcome in and of itself.

The USA has a triple aim of improving the healthcare system: improving the patient experience of care, improving the health of populations and reducing per capita costs of healthcare. ${ }^{41} \mathrm{DAH}_{30}$ seems to be useful, generic metric in this regard. ${ }^{42} \mathrm{DAH}_{30}$ is a measure of the overall burden of care, both in hospital and postdischarge. The perceived success of a hospital discharge plan as perceived by the patient and their principal carer depends on clear communication and meeting expectations. ${ }^{43} \mathrm{DAH}_{30}$ offers transparency and opportunities for benchmarking performance, both of which are important components of quality improvement. ${ }^{13}$ It may influence alternative payment contracts for hospitals.

Postoperative complications add to hospital costs and increase length of stay. ${ }^{44}$ Higher episode payments at 'lowerquality' hospitals have been attributed to higher rates of complications, 30-day readmissions and postdischarge ancillary care. ${ }^{44}$ Serious postoperative complications are both strongly associated with readmission, ${ }^{517}$ increasing the risk by 6.7 -fold, and loss of independence. ${ }^{5}$ Readmission is a frequent, costly and sometimes life-threatening event that is associated with gaps in follow-up care..$^{15} 1745$ Readmission after surgery is thus an established quality 
Table $2 \mathrm{DAH}_{30}$ according to patient and perioperative characteristics

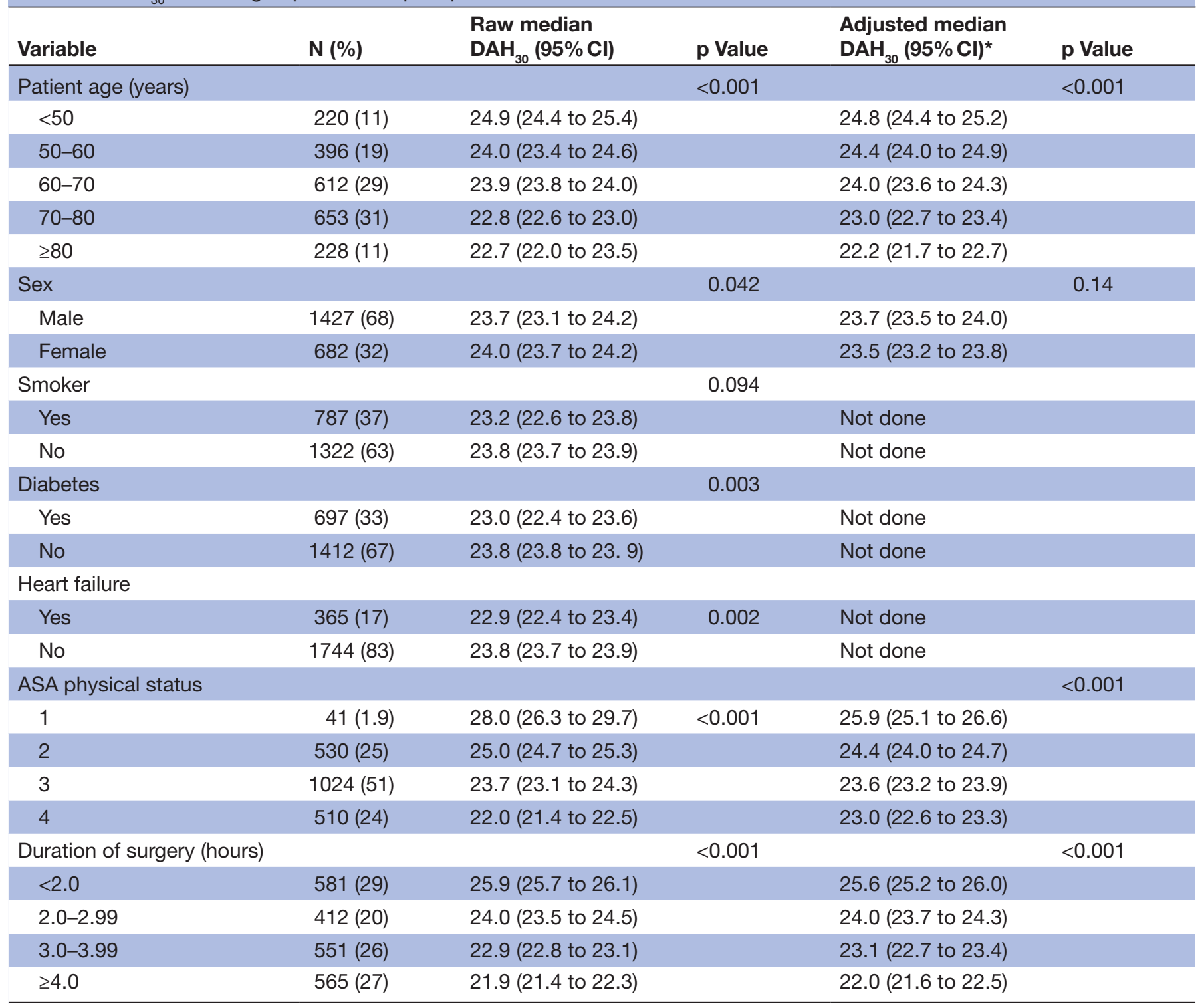

${ }^{*}$ Covariates included in the multivariable adjustment were patient age, sex, ASA physical status and duration of surgery. ASA, American Society of Anesthesiologists; $\mathrm{DAH}_{30}$, days at home up to 30 days after surgery.

Table 3 Median $(95 \% \mathrm{Cl})$ days at home up to 30 days after surgery according to postoperative complications

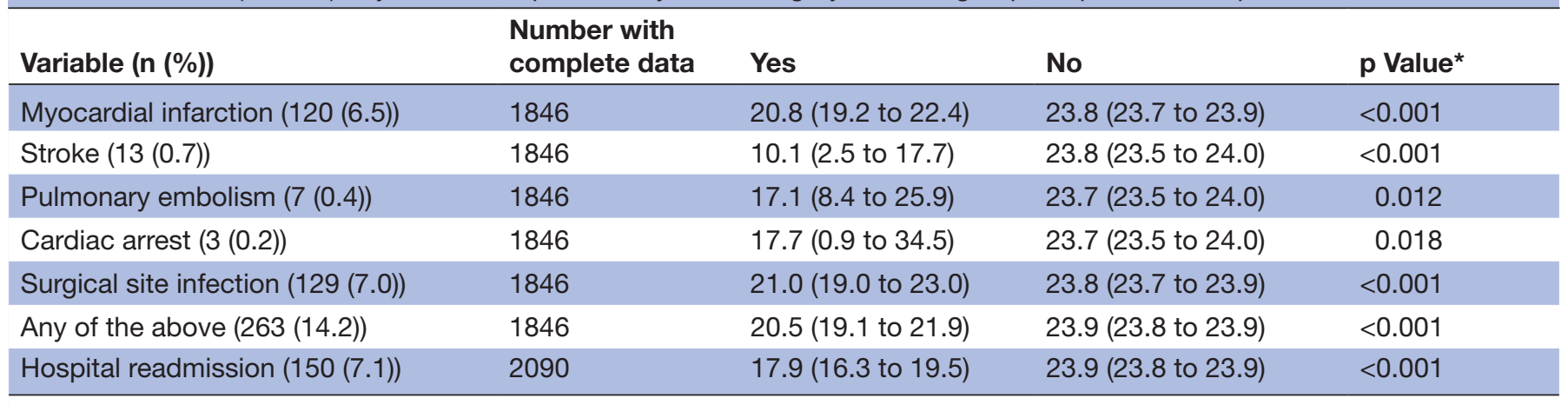

${ }^{*} p$ Values calculated using the quasilikelihood ratio test. 
indicator. Trends in readmissions suggest that US hospitals are responding to incentives to reduce readmissions under the Affordable Care Act. ${ }^{46}{ }^{47}$ Hospital readmission rates are not highly correlated with mortality rates, ${ }^{48}$ so they offer an independent and more sensitive measure of quality. Even though some readmissions are due to chronic medical conditions, ${ }^{45}$ optimal perioperative care should keep these to a minimum and such improvements should be reflected in more $\mathrm{DAH}_{30}$.

Enhanced recovery after surgery programme is designed to reduce complications and shorten length of stay. But this sometimes comes at the cost of increased hospital readmissions. ${ }^{49}{ }^{50}$ The measurement and reporting of $\mathrm{DAH}_{30}$ would identify this and hopefully encourage further quality improvement. Planned discharge to a rehabilitation facility sometimes forms part of an enhanced recovery pathway, and in any case may not be seen by the patient or their family as indicating a poor outcome. Therefore, calculation of $\mathrm{DAH}_{30}$ in some studies could incorporate days spent in a rehabilitation facility as equivalent to being home. In contrast, unplanned admission to a rehabilitation facility would indicate poor care or adverse outcome, and this should be retained in the calculation of $\mathrm{DAH}_{30}$. Care should be taken to avoid missing out-of-network hospitalisations, particularly if relying on hospital system electronic medical records. The latter will otherwise enhance the efficiency of data collection.

Composite endpoints used in perioperative trials are often flawed, ${ }^{51-53}$ typically used to increase the number of events in order to enhance statistical power. $\mathrm{DAH}_{30}$, as a numerical patient-centred measure, provides more statistical power, can be reliably measured and has direct patient-centredness. Although some postoperative complications and poor survival can manifest many months after surgery in those recovering from major surgery or critical illness, ${ }^{29} 5455$ extending measurement out to 90 days after surgery (ie, $\mathrm{DAH}_{90}$ ) may not necessarily provide new or different information because the extra burden and costs of further data collection may outweigh the benefits of the extra information obtained. In addition, disease progression or other aspects of life may confound outcome evaluation of perioperative care.

Our study has several limitations. First, this was a singlecentre study of clinical trial data collected for other purposes; external validity needs to be further evaluated. Second, postoperative in-hospital deaths have a major influence on the calculation of $\mathrm{DAH}_{30}$; this is arguably appropriate because perioperative studies should weight this as the most extreme adverse outcome. More sophisticated modelling could jointly model the risk of death and $\mathrm{DAH}_{30}$ in those discharged alive, and such modelling would be particularly important if the in-hospital mortality rate is moderate or greater. Third, different healthcare settings can be expected to have varied casemix and hospital discharge processes, and hospital discharge may be delayed because of social and process issues unrelated to complications or quality of care. $\mathrm{DAH}_{30}$ should therefore be risk adjusted ${ }^{56}$ Fourth, $\mathrm{DAH}_{30}$ does not provide specific information on which aspects of in-hospital or postdischarge management influences where patients reside after hospitalisation, or the postdischarge use and effectiveness of family physician or other healthcare resources. Fifth, $\mathrm{DAH}_{30}$ is an overall measure of recovery profile and does not inform us about specific complications, level of functioning or well-being. Such aspects should also be included when conducting outcome studies. Sixth, obtaining accurate data on days spent in a rehabilitation facility relies on further follow-up or accurate electronic records. Future studies using $\mathrm{DAH}_{30}$ should prospectively plan to reliably obtain such data.

\section{CONCLUSIONS}

$\mathrm{DAH}_{30}$ has construct validity and is a readily obtainable, generic, patient-centred outcome measure that can better inform patients and physicians when planning surgery. It is a suitable outcome measure for both quality improvement and perioperative clinical trials. $\mathrm{DAH}_{30}$ accounts for prolonged hospital stay, discharge to any postacute care nursing facility, rehospitalisations and early deaths. It thus captures much of the patient-centred experience, and will be maximal when effective and efficient care is achieved.

Acknowledgements The authors would like to thank the study participants for their interest and preparedness to consent to the original trials. PSM is supported in part by Practitioner Fellowships from the Australian National Health and Medical Research Council (NHMRC).

Contributors PSM designed the study, oversaw the ethics application, devised the statistical analysis plan, and drafted and revised the paper. He is the guarantor. MAS, DRM and SM provided intellectual input into the study design, and drafted and revised the paper. SH analysed the data and drafted and revised the paper. SW prepared the ethics application, monitored the data entry and checking, and drafted and revised the paper. IS retrieved and entered all study data, and revised the paper. AF contributed to the statistical analysis, and drafted and revised the paper.

Funding This work was supported by the Australian NHMRC, the New Zealand Health Research Council, and the Australian and New Zealand College of Anaesthetists, and Monash University (Melbourne, Australia).

Disclaimer PSM affirms that this manuscript is an honest, accurate and transparent account of the study being reported; that no important aspects of the study have been omitted; and that any discrepancies from the study as planned have been explained.

Competing interests None declared.

Ethics approval This trial has been approved by the Ethics Committee of Alfred Health, Melbourne, Australia.

Provenance and peer review Not commissioned; externally peer reviewed.

Data sharing statement No additional data available.

Open Access This is an Open Access article distributed in accordance with the Creative Commons Attribution Non Commercial (CC BY-NC 4.0) license, which permits others to distribute, remix, adapt, build upon this work non-commercially, and license their derivative works on different terms, provided the original work is properly cited and the use is non-commercial. See: http://creativecommons.org/ licenses/by-nc/4.0/

(C) Article author(s) (or their employer(s) unless otherwise stated in the text of the article) 2017. All rights reserved. No commercial use is permitted unless otherwise expressly granted.

\section{REFERENCES}

1. Austin JM, McGlynn EA, Pronovost PJ. Fostering transparency in outcomes, quality, safety, and costs. JAMA 2016;316:1661-2. 
2. Lynn J, McKethan A, Jha AK. Value-based payments require valuing what matters to patients. JAMA 2015;314:1445-6.

3. Porter ME. What is value in health care? N Engl $J$ Med 2010;363:2477-81.

4. Lavallee DC, Chenok KE, Love RM, et al. Incorporating patientreported outcomes into health care to engage patients and enhance care. Health Aff 2016;35:575-82.

5. Berian JR, Mohanty S, Ko CY, et al. Association of loss of independence with readmission and death after discharge in older patients after surgical procedures. JAMA Surg 2016;151:e161689.

6. Fried TR, Bradley EH, Towle VR, et al. Understanding the treatment preferences of seriously ill patients. N Engl J Med 2002;346:1061-6.

7. Goldfarb M, Drudi L, Almohammadi M, et al. Outcome reporting in cardiac surgery trials: systematic review and critical appraisal. J Am Heart Assoc 2015;4:e002204.

8. Chalmers I, Clarke M. Outcomes that matter to patients in tombstone trials. Lancet 2001;358:1649.

9. Ariti CA, Cleland JG, Pocock SJ, et al. Days alive and out of hospital and the patient journey in patients with heart failure: insights from the candesartan in heart failure: assessment of reduction in mortality and morbidity (CHARM) program. Am Heart $J$ 2011;162:900-6.

10. Wasywich CA, Gamble GD, Whalley GA, et al. Understanding changing patterns of survival and hospitalization for heart failure over two decades in New Zealand: utility of 'days alive and out of hospital' from epidemiological data. Eur J Heart Fail 2010;12:462-8.

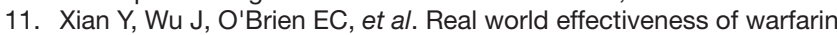
among ischemic stroke patients with atrial fibrillation: observational analysis from patient-centered research into outcomes stroke patients prefer and effectiveness research (PROSPER) study. BMJ 2015;351:h3786.

12. Ellis $G$, Whitehead MA, Robinson D, et al. Comprehensive geriatric assessment for older adults admitted to hospital: meta-analysis of randomised controlled trials. BMJ 2011;343:d6553.

13. Hyder JA, Hirschberg RE, Nguyen LL. Home discharge as a performance metric for surgery. JAMA Surg 2015;150:96-7.

14. Hyder JA, Wakeam E, Habermann EB, et al. Derivation and validation of a simple calculator to predict home discharge after surgery. J Am Coll Surg 2014;218:226-36.

15. Merkow RP, Ju MH, Chung JW, et al. Underlying reasons associated with hospital readmission following surgery in the United States. JAMA 2015;313:483-95.

16. Boney $\mathrm{O}$, Bell M, Bell N, et al. Identifying research priorities in anaesthesia and perioperative care: final report of the joint National Institute of Academic Anaesthesia/James Lind Alliance Research Priority Setting Partnership. BMJ Open 2015;5:e010006.

17. Hinami K, Bilimoria KY, Kallas PG, et al. Patient experiences after hospitalizations for elective surgery. Am J Surg 2014;207:855-62.

18. Burns KE, Jacob SK, Aguirre V, et al. Stakeholder engagement in trial design: survey of visitors to critically III patients regarding preferences for outcomes and treatment options during weaning from mechanical ventilation. Ann Am Thorac Soc 2016:13:1962-8.

19. Hannah D, Lindholm B, Maisch L. Certain uncertainty: life after stroke from the patient's perspective. Circ Cardiovasc Qual Outcomes 2014;7:968-9.

20. Xian Y, O'Brien EC, Fonarow GC, et al. Patient-centered research into outcomes stroke patients prefer and Effectiveness Research: implementing the patient-driven research paradigm to aid decision making in stroke care. Am Heart $J$ 2015;170:36-45.

21. von Elm E, Altman DG, Egger M, et al. The strengthening the reporting of observational studies in epidemiology (STROBE) statement: guidelines for reporting observational studies. Lancet 2007:370:1453-7.

22. Myles PS, Leslie K, Chan MT, et al. The safety of addition of nitrous oxide to general anaesthesia in at-risk patients having major noncardiac surgery (ENIGMA-II): a randomised, single-blind trial. Lancet 2014;384:1446-54

23. Myles PS, Smith JA, Forbes A, et al. Stopping vs. continuing aspirin before coronary artery surgery. N Engl J Med 2016;374:728-37.

24. Myles PS, Smith JA, Forbes A, et al. Tranexamic acid in coronary artery surgery. N Engl J Med 2017;2017:136-48.

25. Thompson EG, Gower ST, Beilby DS, et al. Enhanced recovery after surgery program for elective abdominal surgery at three victorian hospitals. Anaesth Intensive Care 2012;40:450-9.

26. Christelis N, Wallace S, Sage CE, et al. An enhanced recovery after surgery program for hip and knee arthroplasty. Med J Aust 2015;202:363-8.

27. Glassford NJ, Myles P, Bellomo R. The Australian approach to perioperative fluid balance. Curr Opin Anaesthesiol 2012;25:102-10.
28. Vijay A, Grover A, Coulson TG, et al. Perioperative management of patients treated with angiotensin-converting enzyme inhibitors and angiotensin II receptor blockers: a quality improvement audit. Anaesth Intensive Care 2016;44:346-52.

29. Shulman MA, Myles PS, Chan MT, et al. Measurement of disabilityfree survival after surgery. Anesthesiology 2015;122:524-36.

30. Mangram AJ, Horan TC, Pearson ML, et al. Guideline for prevention of surgical site infection, 1999. Infect Control Hosp Epidemiol 1999;20:247-80.

31. Degnim AC, Throckmorton AD, Boostrom SY, et al. Surgical site infection after breast surgery: impact of 2010 CDC reporting guidelines. Ann Surg Oncol 2012;19:4099-103.

32. Thygesen K, Alpert JS, Jaffe AS, et al. Third universal definition of myocardial infarction. Circulation 2012;126:2020-35.

33. Leonardi S, Armstrong PW, Schulte PJ, et al. Implementation of standardized assessment and reporting of myocardial infarction in contemporary randomized controlled trials: a systematic review. Eur Heart J 2013;34:894-902.

34 Koenker R, Bassett G. Regression quantiles. Econometrica 1978;46:33-50.

35 Jackknife WCFJ. Bootstrap and other resampling methods in regression analysis. Ann Stat 1986;14:1261-350.

$36 \mathrm{He}$ X, Hu F. Markov chain marginal bootstrap. J Am Stat Assoc 2002;97:783-95.

37 Koenker R, Machado JAF. Goodness of fit and related inference processes for quantile regression. J Am Stat Assoc 1999;94:1296-310

38 Gornall BF, Myles PS, Smith CL, et al. Measurement of quality of recovery using the QoR-40: a quantitative systematic review. $\mathrm{Br} J$ Anaesth 2013;111:161-9.

39 Liu VX, Rosas E, Hwang J, et al. Enhanced recovery after surgery program implementation in 2 surgical populations in an integrated health care delivery system. JAMA Surg 2017;152:e171032.

40 Yadla S, Ghobrial GM, Campbell PG, et al. Identification of complications that have a significant effect on length of stay after spine surgery and predictive value of 90 -day readmission rate. $J$ Neurosurg Spine 2015;23:807-11.

41 Berwick DM, Nolan TW, Whittington J. The triple aim: care, health, and cost. Health Aff 2008;27:759-69.

42 Lee VS, Kawamoto K, Hess R, et al. Implementation of a value-driven outcomes program to identify high variability in clinical costs and outcomes and association with reduced cost and improved quality. JAMA 2016;316:1061-72.

43 Bauer M, Fitzgerald L, Haesler E, et al. Hospital discharge planning for frail older people and their family. are we delivering best practice? a review of the evidence. J Clin Nurs 2009;18:2539-46.

44 Birkmeyer JD, Gust C, Dimick JB, et al. Hospital quality and the cost of inpatient surgery in the United States. Ann Surg 2012;255:1-5.

45 Jencks SF, Williams MV, Coleman EA. Rehospitalizations among patients in the medicare fee-for-service program. N Engl J Med 2009;360:1418-28.

46 Zuckerman RB, Sheingold SH, Orav EJ, et al. Readmissions, observation, and the hospital readmissions reduction program. $N$ Engl J Med 2016;374:1543-51.

47 Danino JF, Taylor T, Metcalfe CW, et al. Readmission rates and financial penalties after ear, nose and throat surgery: how can we improve? Br J Hosp Med 2015;76:655-7.

48 Hannan EL, Zhong Y, Lahey SJ, et al. 30-day readmissions after coronary artery bypass graft surgery in New York state. JACC Cardiovasc Interv 2011;4:569-76.

49 Ahmed J, Khan S, Lim M, et al. Enhanced recovery after surgery protocols - compliance and variations in practice during routine colorectal surgery. Colorectal Dis 2012;14:1045-51.

50 ERAS Compliance Group. The impact of Enhanced Recovery Protocol Compliance on Elective Colorectal Cancer Resection: results from an international registry. Ann Surg 2015;261:1153-9.

51 Myles PS, Devereaux PJ. Pros and cons of composite endpoints in anesthesia trials. Anesthesiology 2010;113:776-8.

52 Ferreira-Gonzalez I, Permanyer-Miralda G, Busse JW, et al. Composite outcomes can distort the nature and magnitude of treatment benefits in clinical trials. Ann Intern Med 2009;150:566-7.

53 Pogue J, Thabane L, Devereaux PJ, et al. Testing for heterogeneity among the components of a binary composite outcome in a clinical trial. BMC Med Res Methodol 2010;10:49.

54 Khuri SF, Henderson WG, DePalma RG, et al. Determinants of long-term survival after major surgery and the adverse effect of postoperative complications. Ann Surg 2005;242:326-41.

55 Myles PS, Peyton P, Silbert B, et al. Perioperative epidural analgesia for major abdominal surgery for cancer and recurrence-free survival: randomised trial. BMJ 2011;342:d1491. 
hospital readmission rates and differing penalties between safety-net 